\title{
Role of denosumab in the management of skeletal complications in patients with bone metastases from solid tumors
}

This article was published in the following Dove Press journal:

Biologics:Targets and Therapy

II April 2012

Number of times this article has been viewed

\author{
Ursa Brown-Glaberman \\ Alison T Stopeck \\ University of Arizona Cancer Center, \\ Tucson, AZ, USA
}

\begin{abstract}
Skeletal-related events (SREs) including pain, fractures, and hypercalcemia are a major source of morbidity for cancer patients with bone metastases. The receptor activator of NF- $\mathrm{KB}$ ligand (RANKL) is a key mediator of osteoclast formation and activity in normal bone physiology as well as cancer-induced bone resorption. The first commercially available drug that specifically targets and inhibits the RANKL pathway is denosumab, a fully human monoclonal antibody that binds and neutralizes RANKL, thereby inhibiting osteoclast function. In this review, we summarize the major studies leading to the US Food and Drug Administrationapproval of denosumab for the prevention of SREs in patients with bone metastases from solid tumors. Further, we discuss the role of denosumab in the prevention and treatment of SREs and bone loss in cancer patients. As a monoclonal antibody, denosumab has several advantages over bisphosphonates, including improved efficacy, better tolerability, and the convenience of administration by subcutaneous injection. In addition, as denosumab has no known renal toxicity, it may be the preferred choice over bisphosphonates in patients with baseline renal insufficiency or receiving nephrotoxic therapies. However, other toxicities, including osteonecrosis of the jaw and hypocalcemia, appear to be class effects of agents that potently inhibit osteoclast activity and are associated with both denosumab and bisphosphonate use. The data presented highlight the differences associated with intravenous bisphosphonate and denosumab use as well as confirm the essential role bone-modifying agents play in maintaining the quality of life for patients with bone metastases.
\end{abstract}

Keywords: denosumab, bone metastases, solid tumor, breast cancer, prostate cancer, skeletal related events, skeletal complications

\section{Introduction}

Bone is the most common site of metastatic disease in patients with solid tumors. Approximately $30 \%-40 \%$ of patients with lung cancer and $20 \%-25 \%$ of patients with advanced renal cell carcinoma are found to have bone metastasis on autopsy. ${ }^{1}$ In patients with metastatic breast and prostate cancer, $65 \%-80 \%$ will develop bone metastasis, and bone is the most common site of first recurrence in breast cancer patients. ${ }^{1,2}$ In addition, pain from bone metastasis is the most frequent form of pain reported in patients with cancer. ${ }^{3}$ Patients with metastatic disease of the bone are at risk for skeletal-related events, which are defined as: radiation therapy to alleviate pain or prevent fracture, surgery to prevent or treat fracture, pathologic fracture (excluding major trauma), spinal cord compression, and other complications related to skeletal involvement, including hypercalcemia of malignancy. Skeletal-related events are thus a major source of morbidity for cancer patients.
Correspondence: Alison Stopeck University of Arizona Cancer Center I5I 5 N Campbell Avenue, Tucson, AZ 85724, USA

$\mathrm{Tel}+\mathrm{I} 5206262816$

Email astopeck@azcc.arizona.edu 
Cancer patients often experience multiple skeletalrelated events over the course of their disease. Patients with metastatic breast cancer experience an average of four skeletal-related events, lung cancer patients approximately 2.7 skeletal-related events, and prostate cancer patients an average 1.5 skeletal-related events over the course of their disease if untreated. ${ }^{4-6}$ Skeletal-related events also add considerable costs to the management of patients with bone metastases and can be a major cause of hospitalization. Fractures are the most commonly reported skeletal-related events and occur in up to $60 \%$ of untreated breast cancer patients with bone metastasis. ${ }^{7}$ Bone metastases cause pain, limited mobility, fears of future fracture, and loss of function, significantly decreasing quality of life. Thus, the prevention of skeletal-related events in patients with metastatic solid tumors is a vital component of their oncologic care.

Since the 1990s, bisphosphonates have been the mainstay of treatment to prevent skeletal-related events in patients with cancer metastases to bone. Bisphosphonates are rapidly incorporated into bone and are released during osteoclastmediated bone resorption, where they impair the ability of osteoclasts to adhere to the bony surface and inhibit continued bone resorption. Bisphosphonates also decrease osteoclast progenitor development and recruitment, and induce osteoclast apoptosis. The most commonly used intravenous bisphosphonates are zoledronic acid (Zometa ${ }^{\circledR}$, Novartis Pharmaceuticals East Hanover, NJ) and pamidronate (Aredia ${ }^{\circledR}$, Novartis Pharmaceuticals East Hanover, NJ).

Zoledronic acid is the most potent bisphosphonate currently available, 500-1000 times more potent than pamidronate, and is the first drug in this class approved for use in all solid tumor patients with bone metastases as well as in multiple myeloma. Its use has been studied most extensively in breast cancer, prostate cancer, multiple myeloma, and lung cancer. ${ }^{4,5,8-11}$ Despite optimal bisphosphonate therapy, $30 \%-50 \%$ of cancer patients with bone metastases still develop skeletal-related events while on bisphosphonate therapy. ${ }^{5,8}$ In addition, there are concerning treatment-related side effects associated with bisphosphonates, including gastrointestinal irritation, nephrotoxicity, osteonecrosis of the jaw, and hypocalcemia. Intravenous infusion of zoledronic acid can be associated with an acute-phase reaction, including bone pain, fever, and chills in up to $30 \%$ of patients following their first infusion. In addition, intravenous bisphosphonates, and in particular zoledronic acid carry the risk of renal toxicity that is dose-dependent and infusion time-dependent. Zoledronic acid is currently not recommended for use in patients with a creatinine clearance lower than $30 \mathrm{~mL} /$ minute and must be dose-reduced if creatinine clearance is less than $60 \mathrm{~mL} /$ minute. $^{12}$ Consequently, monthly monitoring of renal function is required prior to each dose of intravenous bisphosphonate. The use of bisphosphonates in patients with solid tumors and renal dysfunction is thus limited and the concomitant use of zoledronic acid with other nephrotoxic drugs or chemotherapeutic agents can be problematic.

Receptor activator of NF- $\kappa \mathrm{B}$ ligand (RANKL)-mediated osteoclast activity is a key regulator of bone metabolism. RANKL is produced by osteoblasts, bone marrow stromal cells, and other cells under the control of various proresorptive growth factors, hormones, and cytokines. Osteoblasts and stromal cells produce osteoprotegerin, a decoy receptor, which binds to and inactivates RANKL. In the absence of osteoprotegerin, RANKL activates the RANK receptor, which is found on osteoclasts and preosteoclast precursors. RANK-RANKL interactions lead to preosteoclast recruitment, fusion into multinucleated osteoclasts, osteoclast activation, and osteoclast survival. The pivotal role of this pathway in bone metabolism make it a prime target for the treatment of bone disease. The first commercially available drug to target the RANK-RANKL pathway is denosumab, a fully human monoclonal antibody that specifically binds and neutralizes RANKL, thereby inhibiting osteoclast function. The initial Phase I trials demonstrated that osteoclastic activity is almost completely eradicated while denosumab is in circulation. ${ }^{13}$ However, the effect is reversible, as indicated by a rise in markers of bone turnover when the drug is cleared. ${ }^{13}$

Initial trials with denosumab were in the treatment of women with primary osteoporosis. In the Phase III FREEDOM trial, 7868 postmenopausal women (aged 60-90 years) with osteoporosis were randomly assigned to subcutaneous denosumab (60 mg every 6 months) or placebo. ${ }^{14}$ After 3 years, denosumab improved bone mineral density compared with placebo. In addition, biochemical markers of bone turnover and fractures were significantly reduced in patients taking denosumab. ${ }^{14}$ When compared with bisphosphonates in the treatment of primary osteoporosis, denosumab has shown improvements in both bone mineral density and markers of bone loss. ${ }^{15}$

Denosumab has also been studied in bone loss associated with hormonal therapy in both breast and prostate cancer. In postmenopausal patients with hormone receptor-positive breast cancer, aromatase inhibitor use is associated with bone loss and increased fracture risk. ${ }^{16}$ Guidelines from the American Society of Clinical Oncology and the National Comprehensive Cancer Network recommend bone mineral 
density testing with a dual-emission x-ray absorptiometry scan for postmenopausal women taking aromatase inhibitors and drug therapy for those with documented osteoporosis. ${ }^{17,18}$ In a randomized, double-blind, placebo-controlled trial, 252 women with hormone receptor-positive, early-stage breast cancer treated with adjuvant aromatase inhibitor therapy were randomly assigned to receive placebo or subcutaneous denosumab $60 \mathrm{mg}$ every 6 months. ${ }^{19}$ At enrollment, all patients were required to have evidence of low bone mass (excluding osteoporosis). At 12 and 24 months, lumbar spine bone mineral density increased by $5.5 \%$ and $7.6 \%$, respectively, in the denosumab group compared with the placebo group $(P=0.0001)$. In the HALT (Hormone Ablation Bone Loss) trial, 1468 men receiving androgen deprivation therapy for nonmetastatic prostate cancer were randomly assigned to denosumab (60 mg subcutaneously every 6 months) or placebo. ${ }^{20}$ Eligibility included male gender, age $\geq 70$ years or $<70$ years with baseline low bone mineral density ( $\mathrm{T}$ score at the lumbar spine, total hip, or femoral neck of less than -1.0). At 24 months, denosumab was associated with increased bone mineral density at all measured sites, including the total hip, femoral neck, distal third of the radius, and whole body (absolute difference versus placebo of $4.8 \%, 3.9 \%, 5.5 \%$, and $4.0 \%$, respectively, $P<0.001$ for all comparisons). Because of the increased power of this larger study, a statistically significant decrease in new vertebral fractures at 36 months was also observed in the denosumab arm (1.5\% versus $3.9 \%$ with placebo, relative risk, $0.38 ; 95 \%$ confidence interval, $0.19-0.78 ; P=0.006$ ). The promising outcomes in the initial trials with denosumab in treatment-related osteoporosis associated with breast and prostate cancer led to exploration of its use for the prevention of skeletal-related events in patients with solid tumors and bone metastasis, which is the focus of this review.

\section{Dosing and side effects}

Denosumab has US Food and Drug Administration (FDA) approval under two brand names. Under the brand name Prolia ${ }^{\circledR}$ (Amgen Inc, Thousand Oaks, CA), it is indicated for the treatment of primary osteoporosis in postmenopausal women at a dose of $60 \mathrm{mg}$ subcutaneously every 6 months. This dose is also FDA-approved for the treatment of bone loss associated with aromatase inhibitor therapy in early-stage breast cancer and androgen deprivation therapy for nonmetastatic prostate cancer. Under the brand name Xgeva ${ }^{\circledR}$ (Amgen Inc), denosumab is FDA-approved for the prevention of skeletal-related events in patients with bone metastases from solid tumors at a dose of $120 \mathrm{mg}$ subcutaneously every four weeks.
Denosumab absorption is rapid and sustained, with a bioavailability of $62 \%$, a steady-state mean serum concentration of $20.5 \mu \mathrm{g} / \mathrm{mL}$, and an elimination half-life of 28 days. $^{21} \mathrm{~A}$ decrease in bone resorption markers is observed within 24 hours after initial dose administration, and steady-state levels are achieved by 6 months following multiple doses at the $120 \mathrm{mg}$ monthly schedule. ${ }^{13,21}$ The initial Phase I trial in healthy postmenopausal women demonstrated that a single denosumab dose of $3.0 \mathrm{mg} / \mathrm{kg}$ could suppress markers of bone turnover, including urinary collagen type 1 crosslinked N-telopeptide (NTx), by up to $80 \%$ for a duration of several months. ${ }^{13}$ In a Phase II trial of patients with bone metastases from solid tumors treated with denosumab, doses of 30-180 mg administered every 4 or 12 weeks were compared with monthly intravenous bisphosphonate therapy in patients with elevated urinary NTx/creatinine levels despite intravenous bisphosphonate therapy. ${ }^{22}$ Patients treated with denosumab showed a significant decline in urinary $\mathrm{NTx} /$ creatinine ratio compared with patients who continued therapy with intravenous bisphosphonates, suggesting that denosumab may be superior to intravenous bisphosphonates at suppressing bone resorption. ${ }^{22}$ A second Phase II trial in patients with metastatic breast cancer treated with multiple doses and schedules of denosumab also confirmed a greater reduction in urinary NTx/creatinine levels in denosumabtreated patients compared with intravenous bisphosphonatetreated patients $(74 \%$ versus $63 \%){ }^{23}$

Denosumab elimination is thought to occur through the immunoglobulin clearance pathway via the reticuloendothelial system, similar to that of other monoclonal antibodies and is thus thought to be independent of renal or hepatic function. ${ }^{24}$ The incidence of adverse events related to renal toxicity observed in the registration trials for preventing skeletal-related events in patients with bone metastases was lower in the denosumab arm than in the zoledronic acid arm and similar to the rate seen in the observational arm of prior bisphosphonate trials. The three larger Phase III registration trials comparing denosumab with zoledronic acid are discussed in further detail below. Dose reductions and renal monitoring are not required with denosumab therapy. However, there is a lack of safety data in patients with severe renal dysfunction because patients with creatinine clearance levels less than $30 \mathrm{~mL} /$ minute were excluded from the Phase III trials because the comparator, zoledronic acid, is contraindicated in this patient population. In the one small trial in which denosumab was administered as a single $60 \mathrm{mg}$ dose to 55 patients, including 17 patients with severe chronic renal disease (creatinine clearance $\leq 30 \mathrm{~mL} /$ minute) 
or requiring hemodialysis, pharmacokinetics, and changes in biomarkers of bone resorption was unaffected by renal function. However, patients with severe chronic renal disease were at a greater risk of severe hypocalcemia with denosumab compared with patients with normal renal function. ${ }^{21}$ The risk of hypocalcemia at the dosing schedule used in the setting of bone metastasis (120 mg every 4 weeks) has not been adequately evaluated in patients with a creatinine clearance of less than $30 \mathrm{~mL} / \mathrm{minute}$ or in those who are receiving dialysis. More frequent monitoring of serum calcium levels as well as ensuring adequate vitamin $\mathrm{D}$ levels prior to and upon initiation of denosumab therapy seems prudent in this population until additional safety studies are completed. The drug has also not been studied in the pediatric population.

In the setting of osteoporosis, the FREEDOM (Fracture Reduction Evaluation of Denosumab in Osteoporosis Every 6 Months) trial is the largest single trial comparing denosumab with placebo for the prevention of fractures. ${ }^{14}$ In this study, there were no significant differences between the 3900 subjects who received denosumab and those who received placebo with regard to the total incidence of adverse events, serious adverse events, or discontinuation of study treatment because of adverse events. ${ }^{14}$ In addition, there was no increase in the risk of cancer, overall rate of infection, cardiovascular disease, delayed fracture healing, or hypocalcemia, and there were no cases of osteonecrosis of the jaw. Neutralizing antibodies against denosumab have not been identified.

Because RANKL is expressed on subsets of T and B cells, there is a theoretical possibility that denosumab may be immunosuppressive. RANKL-deficient mice lack normal lymph node development and have inhibition of early $\mathrm{T}$ and B lymphocyte development. ${ }^{25}$ However, in clinical trials, a statistically significant or clinically meaningful effect on the immune system has not been observed. In one early trial, denosumab therapy had no significant effect on mean white blood cell counts, absolute lymphocyte counts, T and B cell counts, or immunoglobulins, and no meaningful difference was seen regarding incidence of infection. ${ }^{13}$ Phase II and III trials of denosumab for the treatment and prevention of osteoporosis suggested a slight increase in the rate of certain infectious complications, including cellulitis. ${ }^{14,26}$ However, the overall infection rate did not differ from placebo, and an association between denosumab and serious adverse infectious events has not been observed in any of the three large Phase III registration trials in cancer patients. Table 1 summarizes the adverse events observed in the three Phase III trials in patients with breast cancer, prostate cancer, and other solid tumors or multiple myeloma.
Table I Combined data for adverse events from three Phase III registration trials in patients with breast cancer, prostate cancer, and other solid tumors or multiple myeloma ${ }^{38}$

\begin{tabular}{|c|c|c|}
\hline Adverse event & $\begin{array}{l}\text { Denosumab } \\
(n=284 I)^{a}\end{array}$ & $\begin{array}{l}\text { Zoledronic acid } \\
(\mathrm{n}=\mathbf{2 8 3 6})^{\mathrm{a}}\end{array}$ \\
\hline Adverse events & $2734(96.2)$ & $2745(96.8)$ \\
\hline $\begin{array}{l}\text { Adverse events leading } \\
\text { to study discontinuation }\end{array}$ & $270(9.5)$ & $280(9.9)$ \\
\hline $\begin{array}{l}\text { CTCAE Grade 3, } 4 \text {, or } \\
5 \text { adverse events }\end{array}$ & $2000(70.4)$ & $2009(70.8)$ \\
\hline Serious adverse events & $1599(56.3)$ & $1620(57.1)$ \\
\hline Adverse events of inter & & \\
\hline $\begin{array}{l}\text { Acute phase reactions } \\
\text { (first } 3 \text { days) }\end{array}$ & $246(8.7)$ & $572(20.2)$ \\
\hline Hypocalcemia & $273(9.6)$ & $|4|(5.0)$ \\
\hline Osteonecrosis of the jaw & $52(1.8)$ & $37(1.3)$ \\
\hline
\end{tabular}

Note: apatients who received at least one dose of active drug. Abbreviation: CTCAE, Common Terminology Criteria for Adverse Events.

\section{Disease-specific use}

There have been three international Phase III randomized, double-blind, double-dummy, active controlled studies including over 5700 patients comparing denosumab with zoledronic acid for the prevention of skeletal-related events in patients with bone metastases. These three registration trials were of identical design and focused on the prevention of skeletal-related events in patients with breast cancer, prostate cancer, and other solid tumors or multiple myeloma, and led to FDA approval of denosumab for this indication.

\section{Breast cancer}

The first published Phase III trial enrolled 2046 patients with metastatic breast cancer and radiologic evidence of at least one bone metastasis. ${ }^{27}$ Patients with a creatinine clearance $<30 \mathrm{~mL} /$ minute were excluded because zoledronic acid is contraindicated in that patient population. Other exclusion criteria included prior bisphosphonate use for bone metastases and nonhealed dental/oral surgery. Patients were randomly assigned to receive either subcutaneous denosumab at $120 \mathrm{mg}$ and intravenous placebo $(\mathrm{n}=1026)$ or intravenous zoledronic acid $4 \mathrm{mg}$ (with adjustment for creatinine clearance) and subcutaneous placebo $(\mathrm{n}=1020)$ every 4 weeks. Supplementation with calcium $\geq 500 \mathrm{mg}$ and vitamin $\mathrm{D} \geq 400$ IU daily was strongly encouraged. The primary endpoint was time to first skeletal-related event, with the study powered to detect noninferiority of denosumab versus zoledronic acid. Secondary endpoints included time to first on-study skeletal-related event (superiority test) and time to first and subsequent on-study skeletal-related event (multiple event analysis). The majority of patients in 
this study were postmenopausal, with hormone (estrogen/ progesterone) receptor-positive breast cancer. Denosumab delayed the time to first on-study skeletal-related event by $18 \%$ compared with zoledronic acid (hazards ratio [HR] $0.82 ; 95 \%$ confidence interval $[\mathrm{CI}] 0.71-0.95 ; P<0.001$ for noninferiority, $P=0.01$ for superiority). Median time to first skeletal-related event was 26.4 months in the zoledronic acid group and had not yet been reached in the denosumab group at the time of the primary analysis. With an additional 4 months of blinded treatment, the median time to first skeletal-related event was reached in the denosumab arm at 32.4 months. ${ }^{28}$ Denosumab also reduced the risk of subsequent skeletalrelated events by $23 \%$ (risk ratio 0.77 ; 95\% CI $0.66-0.89$, $P=0.001)$. Overall survival and disease progression were similar between the two groups. As in the Phase I and II trials, denosumab treatment resulted in greater suppression of bone turnover markers, including urinary NTx. At week 13, urinary NTx/creatinine levels decreased by $80 \%$ with denosumab and by $68 \%$ with zoledronic acid $(P<0.001)$.

The overall rates of serious adverse events and adverse events, including infectious complications, were similar between the two groups. Most of the observed adverse events were felt to be related to the patient's concurrent chemotherapy and/or advanced malignancy. However, several adverse events were different between the two arms, including the incidence of flu-like symptoms (acute-phase reactions, $27.3 \%$ with zoledronic acid versus $10.4 \%$ with denosumab) and renal toxicity $(8.5 \%$ with zoledronic acid versus $4.9 \%$ with denosumab). No patient treated with denosumab and 10 patients $(1 \%)$ treated with zoledronic acid experienced serious adverse events associated with acute-phase reactions during the first 3 days after treatment. ${ }^{29}$ These events included pyrexia $(n=7)$, bone pain $(n=2)$, and asthenia, back pain, chest pain, chills, headache, and malaise $(n=1$ each). Nine of the 10 patients in the zoledronic acid group with serious acute-phase reactions required hospitalization or prolongation of hospitalization. ${ }^{29}$

Toothache, which was not associated with osteonecrosis of the jaw (5.6\% denosumab versus 3.7\% zoledronic acid) and hypocalcemia (5.5\% denosumab versus $3.4 \%$ zoledronic acid) were more common in the denosumab group. None of the hypocalcemic events were fatal, and the majority required outpatient monitoring and additional oral or intravenous supplementation. Osteonecrosis of the jaw occurred infrequently in both groups and at rates that were not significantly different $(2.0 \%$ in denosumab versus $1.4 \%$ in zoledronic acid, $P=0.39$ ). The majority of patients who developed osteonecrosis of the jaw had known risk factors, including poor oral hygiene and/or prior dental extractions. As with zoledronic acid, the package insert for denosumab recommends that patients should consult a dentist and undergo preventive dentistry (ie, extraction of unsalvageable teeth) prior to initiating therapy with a bone-modifying agent to reduce the risk of osteonecrosis of the jaw. ${ }^{21}$

Patient-reported outcomes analyzed in the trial included pain using the Brief Pain Inventory and quality of life as assessed by the Functional Assessment of Cancer TherapyGeneral (FACT-G) score. ${ }^{30}$ Patients were asked to complete the Brief Pain Inventory at baseline, day 8, and before each monthly visit through the end of study. In patients with scores of no/mild pain at baseline $(n=1042)$, median time to development of moderate/severe pain with denosumab was 295 days compared with 176 days in those treated with zoledronic acid (HR 0.78, 95\% CI 0.67-0.92, $P=0.0024$ ). The proportion of patients with no/mild pain at baseline who reported moderate/severe pain on-study was consistently lower for denosumab than for zoledronic acid through week 73. Time to pain improvement was similar between treatment arms (median 82 days for denosumab, median 85 days for zoledronic acid; HR 1.02, 95\% CI 0.91-1.15, $P=0.7245)$. Similarly, health-related quality of life was higher in the denosumab arm than in the zoledronic acid arm throughout the study, and a greater proportion of denosumabtreated patients reported improvements in emotional and physical well-being. ${ }^{30}$

Based on the results of this trial and the FDA approval of denosumab for this indication, both the American Society of Clinical Oncology and National Comprehensive Cancer Network guidelines were updated to include therapy with denosumab at $120 \mathrm{mg}$ subcutaneously every 4 weeks as a recommended regimen for preventing skeletal-related events in patients with bone metastases from breast cancer. ${ }^{18,31}$

\section{Prostate cancer}

The Phase III trial in prostate cancer randomized 1904 patients with metastatic castrate-resistant prostate cancer to either denosumab or zoledronic acid treatment using an identical double-blind, double-dummy, active-controlled design. ${ }^{32}$ While skeletal lesions in prostate cancer are generally described as osteoblastic, osteoclast activity is known to be increased and to play an essential role. Patients with bone metastases from prostate cancer and high urinary NTx levels have an increased risk of skeletal-related events, time to a first skeletal-related event, disease progression, and death. ${ }^{33}$ In the Phase III trial, eligible patients had histologically confirmed prostate cancer, evidence of at least one bone 
metastases, and documented failure of at least one hormonal therapy. Exclusion criteria included current or previous treatment with bisphosphonates for bone metastases, planned radiation therapy or surgery to bone, life expectancy less than 6 months, current or previous osteonecrosis of the jaw, planned invasive dental procedures, and malignancy other than prostate cancer. Patients were strongly encouraged to take vitamin $\mathrm{D}$ and calcium supplementation. As in the breast cancer trial, patients were required to have a creatinine clearance $>30 \mathrm{~mL} / \mathrm{minute}$ to be eligible for randomization to the zoledronic acid arm.

At the time of the primary analysis, patients had been on study for an average of 12.2 months in the denosumab group and 11.2 months in the zoledronic acid group. ${ }^{32}$ In the zoledronic acid arm, 22\% of patients required dose adjustment for renal function and $15 \%$ of patients required doses to be held secondary to decreased renal function. In the denosumab arm, doses were not held or adjusted for renal function. Median time to first on-study skeletalrelated event was 20.7 months for patients on denosumab (95\% CI 18.8-24.9) versus 17.1 months for those on zoledronic acid (95\% CI 15.0-19.4), with an HR of $0.82(95 \%$ CI $0.71-0.95, P=0.002$ for noninferiority and $P=0.008$ for superiority). This corresponds to a delay in time to first on-study skeletal-related event of $18 \%$ with denosumab compared with zoledronic acid, identical to the results from the similarly designed and powered trial in metastatic breast cancer. Denosumab also significantly delayed the time to first and subsequent on-study skeletal-related event (HR 0.82, 95\% CI 0.72-0.94, $P=0.004)$. Overall survival and disease progression were not significantly different between the treatment groups. At week 13, the decrease in urinary NTx/ creatinine was significantly greater in the denosumab group (median decrease of $84 \%$ in the denosumab group versus $69 \%$ in the zoledronic acid group, $P<0.0001)$. No neutralizing antibodies to denosumab were detected.

Overall, occurrence of adverse events and serious adverse events were similar between the groups. Hypocalcemia was more common in the denosumab group $(13 \%$ in the denosumab group versus $6 \%$ in the zoledronic acid group, $P<0.0001)$. The majority of these cases were isolated events and mild to moderate in severity. None of the episodes of hypocalcemia were fatal. The cumulative rate of osteonecrosis of the jaw between the two groups was not statistically significant, occurring in 1\% (12 patients) in the zoledronic acid group versus 2\% (22 patients) in the denosumab group. As in prior studies, the majority of these patients had a history of tooth extraction, a dental appliance, or poor oral hygiene. There was no difference in the rate of infection between the two groups. Adverse events associated with acute-phase reactions occurred in $8 \%$ of patients on denosumab and $18 \%$ of patients on zoledronic acid. Adverse events related to renal impairment were similar between the two groups, at $15 \%$ in the denosumab group and $16 \%$ in the zoledronic acid group. However, the zoledronic acid group required more frequent dose adjustment and dose-holding for renal dysfunction.

As in the Phase III study in breast cancer patients, the authors of this study concluded that denosumab was superior to zoledronic acid for the prevention of skeletal complications due to metastasis. Denosumab is recommended by the National Comprehensive Cancer Network as an option for the prevention of skeletal-related events in patients with metastatic prostate cancer. ${ }^{34}$

\section{Solid tumors other than breast and prostate cancer}

The third Phase III trial of denosumab in the setting of metastatic disease was carried out in 1776 patients with multiple myeloma or solid tumors other than breast or prostate cancer with at least one bone metastasis or osteolytic lesion. ${ }^{35}$ The study design was identical to that of the other two Phase III trials described previously. Approximately $40 \%$ of enrolled patients had non-small cell lung cancer and $10 \%$ had multiple myeloma. At the time of primary analysis, only $20 \%$ of patients remained on study, with the majority of patients discontinuing therapy as a result of death $(35 \%)$, withdrawal of consent $(15 \%)$, or disease progression $(13 \%)$. This trial had the shortest median time on study at approximately 7 months in both treatment groups.

The median time to first on-study skeletal-related event was 20.6 months for denosumab and 16.3 months for zoledronic acid. Denosumab was noninferior to zoledronic acid in delaying time to first on-study skeletal-related event (HR $0.84,95 \%$ CI $0.71-0.98, P=0.0007$ ), with superiority for denosumab nearing statistical significance at a $P$ value of 0.06 . In this study, denosumab also failed to reduce time to first and subsequent skeletal-related events significantly with an HR of 0.90 for denosumab compared with zoledronic acid (95\% CI 0.77-1.04, $P=0.14$ ). Overall survival and disease progression were similar between the groups. The smaller number of patients randomized and shorter time on study yielded fewer skeletal-related events in this trial compared with the similarly designed breast and prostate trials, and thus may be the reason for the less dramatic improvements with denosumab seen in this study. 
As in prior studies, denosumab resulted in greater suppression of the urinary NTx/creatinine ratio $(76 \%$ decrease in the denosumab groups versus $65 \%$ in those on zoledronic acid, $P \leq 0.001$ ). When stratified by tumor type, the HR for time to first on-study skeletal-related event for denosumab versus zoledronic acid was $0.84(95 \% \mathrm{CI}$ $0.64-1.10, P=0.20$ ) for non-small cell lung cancer and 0.76 for other solid tumors $(95 \%$ CI $0.62-0.99, P=0.04$ ). However, the HR for the 180 patients treated with multiple myeloma was only $1.03(95 \% \mathrm{CI} 0.68-1.57, P=0.89)$ and thus the FDA approval of denosumab is limited to patients with solid tumors and excludes treatment of patients with multiple myeloma. Currently, there is an ongoing large Phase III trial comparing denosumab with zoledronic acid therapy specifically in patients with multiple myeloma. Compared with zoledronic acid, the number needed-to-treat analysis showed that treatment of 9.9 patients with denosumab would prevent one additional first skeletal-related event per patient-year. ${ }^{36}$ As in the Phase III trial in breast cancer patients, pain control was monitored at baseline, day 8 , and before each monthly visit patients by the Brief Pain Inventory. ${ }^{37}$ Patients on denosumab experienced a delay in clinically significant pain worsening compared with patients on zoledronic acid (median 169 days in denosumab, 143 days in zoledronic acid; HR $0.85,95 \%$ CI $0.73-0.98$, $P=0.02$ ).

Patients in both groups experienced similar rates of overall adverse events. ${ }^{35}$ Hypocalcemia occurred more frequently in the denosumab group as compared with the zoledronic acid group ( $10.8 \%$ versus $5.8 \%$ ), including grade 3 or 4 hypocalcemia in 20 patients $(2.3 \%)$ on denosumab and nine patients $(1.0 \%)$ on zoledronic acid. As in prior studies, rates of osteonecrosis of the jaw were similar between the two groups (11 patients [1.3\%] in the zoledronic acid group versus 10 patients [1.1\%] in the denosumab group) and osteonecrosis of the jaw was seen primarily in patients with known risk factors. Acute-phase reactions were more common in the zoledronic acid group (14.4\% zoledronic acid versus $6.9 \%$ denosumab). Dose reduction was required in $17.3 \%$, and $8.9 \%$ of doses were held in patients on zoledronic acid due to renal dysfunction. Despite these dose adjustments, renal dysfunction was more common in the zoledronic acid group $(10.9 \%$ versus $8.3 \%)$.

A meta-analysis of the three registration Phase III trials in patients with bone metastasis was recently presented in abstract form and confirms the consistency of the data. ${ }^{38}$ The effects of denosumab versus zoledronic acid were evaluated with respect to time to first on-study skeletal-related event for noninferiority (primary endpoint) and superiority (secondary endpoint), and time to first and subsequent skeletal-related event (secondary endpoint). A total of 5723 patients were evaluated, 2861 in the zoledronic acid group and 2862 in the denosumab group. In this combined analysis, denosumab significantly prevented or delayed the time to first on-study skeletal-related event or hypercalcemia of malignancy, with a risk reduction of $17 \%$ (HR $0.83,95 \%$ CI $0.76-0.90$, $P<0.001$ for both noninferiority and superiority). The median time to first on-study skeletal-related event was 27.7 months with denosumab versus 19.4 months with zoledronic acid, resulting in a median delay of 8.2 months in favor of denosumab therapy. The effect of denosumab was consistent across all types of skeletal-related events (fracture, radiation, surgery, and spinal cord compression). These data are summarized in Table 2. In addition, combined analysis from the Phase III trials in patients with metastatic breast cancer and in patients with solid tumors other than breast or prostate cancer showed a significant decrease in hypercalcemia of malignancy in those treated with denosumab (HR $0.63,95 \%$ CI $0.41-0.98, P=0.042) .{ }^{39}$ Disease progression and overall survival were similar between treatment groups, as was the incidence of all adverse events and serious adverse events. However, as with the individual trials, there was an increased incidence of hypocalcemia in the denosumab group $(9.6 \%$ versus $5.0 \%)$ and acute-phase reactions $(20.2 \%$ versus $8.7 \%$ ) in the zoledronic acid group (Table 1). Incidence of osteonecrosis of the jaw was infrequent and similar between the treatment groups, with a cumulative incidence of $1.3 \%$

Table 2 Hazard ratios for development of first skeletal-related event by type

\begin{tabular}{ll}
\hline Type of SRE & Hazard ratio $(95 \% \mathrm{CI})$ \\
\hline Any SRE & $0.83(0.76-0.90)$, \\
& $P<0.001$ in favor of denosumab \\
& $0.86(0.76-0.96)$, \\
Pathological fracture & $P=0.090$ in favor of denosumab \\
& $0.77(0.69-0.87)$, \\
Radiation to bone & $P \leq 0.001$ in favor of denosumab \\
& $0.89(0.65-1.21)$, \\
Spinal cord compression & $P=0.45$ in favor of denosumab \\
Surgery to bone & $0.86(0.6 I-1.21)$, \\
& $P=0.38$ in favor of denosumab \\
Hypercalcemia of malignancy & $0.63(0.4 I-0.98)$, \\
& $P=0.042$ in favor of denosumab \\
\hline
\end{tabular}

Notes: Combined data from three Phase III registration trials in patients with breast cancer, prostate cancer, and other solid tumors or multiple myeloma. ${ }^{38,39}$ alncludes data from the two Phase III registration trials in patients with breast cancer, and metastasis from solid tumors other than breast or prostate cancer and multiple myeloma. The metastatic prostate cancer trial was not included because of the low number of events.

Abbreviations: $\mathrm{Cl}$, confidence interval; SRE, skeletal-related events. 
(37 events) in the zoledronic acid group compared with $1.8 \%$ (52 events) in the denosumab group. Further analysis of osteonecrosis of the jaw events suggested poor oral hygiene, dental extractions, and dental appliances accounted for the majority of cases, with up to $40 \%$ of the cases resolving with conservative therapies, including oral and/or topical antibiotics and surgical debridement after a median of 8 months. ${ }^{40}$ Only $4 \%$ of patients required bone resection for refractory disease.

Recently, further safety data have been presented for denosumab use in patients with metastatic breast cancer, including patients on therapy for up to 5 years. ${ }^{41}$ In this analysis of patients from the open-label extension phase of the metastatic breast cancer registration trial, no new safety signals were observed in patients who switched from zoledronic acid to denosumab therapy or who remained on denosumab therapy for up to 5 years (median time on denosumab 19.1 months, range 0.1-59.8 months).

\section{Future uses}

The role of denosumab for the prevention of skeletal-related events in patients with bone metastasis from solid tumors has now been established in three large, well-designed, and definitive trials. Potential clinical questions include indications for and timing of transition from intravenous bisphosphonate to denosumab. Obvious indications for switching to denosumab include progressive renal insufficiency or intolerance to side effects associated with bisphosphonates. The Phase III STAND (Study of Transitioning from AleNdronate to Denosumab) trial looked at sequential use of oral bisphosphonates followed by denosumab in postmenopausal women with primary osteoporosis. ${ }^{42}$ Postmenopausal women at least 55 years of age with lumbar spine or total hip bone mineral density measurements corresponding to a $\mathrm{T}$ score of -2.0 to -4.0 who had been receiving alendronate at $70 \mathrm{mg} /$ week for at least 6 months were eligible. Subjects were randomized to denosumab $60 \mathrm{mg}$ subcutaneously every 6 months versus continuing oral alendronate. All subjects were supplied with calcium and vitamin D supplements daily. The primary efficacy endpoint was the percentage change in total hip bone mineral density after 12 months of therapy. Bone mineral density at the total hip increased significantly more in patients transitioned to denosumab (1.90\%, 95\% CI 1.61\%-2.18\%) compared with patients continuing on alendronate $(1.05 \%$, 95\% CI 0.76\%-1.34\%, $(P<0.0001)$. Sequential use has also been explored in a Phase II trial in which 111 patients with solid tumors and bone metastasis previously treated with bisphosphonate were randomized to continue intravenous bisphosphonates versus switching to subcutaneous denosumab $180 \mathrm{mg}$ subcutaneously every 4 or 12 weeks). ${ }^{22}$ Urinary NTx was reduced to below $50 \mathrm{nmol} / \mathrm{L}$ by week 13 (the primary endpoint) in $71 \%$ of patients on denosumab versus $29 \%$ of patients who continued on intravenous bisphosphonates $(P<0.001)$. The percentage of patients experiencing a first on-study skeletal-related event during the 25 -week treatment period was $8 \%$ in the denosumab arm versus $17 \%$ in the intravenous bisphosphonate arm (odds ratio $0.31 ; 95 \% \mathrm{CI} 0.08-1.18$ ). These trials suggest a role for switching to denosumab in patients who are currently receiving oral or intravenous bisphosphonates and experience a skeletal-related event or who continue to have an elevated urinary NTx level despite bisphosphonate therapy. There are currently no data to support the combined use of a bisphosphonate plus denosumab to reduce osteoclast activity further. Because both agents are extremely potent osteoclast inhibitors, the risk of increased toxicity with combination therapy, especially with regard to osteonecrosis of the jaw development and hypocalcemia, would be concerning.

In addition to preventing skeletal-related events, a current area of active research for bone-modifying agents is in the prevention or delay of cancer progression. Recently, several trials in early-stage breast cancer patients suggest a role for bone-modifying agents in improving disease-free survival. The ABCSG-12 trial in premenopausal women with early-stage breast cancer is perhaps the most definitive of the recent trials, suggesting a role for bone-targeted therapy in the prevention of breast cancer recurrence. ${ }^{43}$ In this trial, patients treated with 3 years of twice-yearly intravenous zoledronic acid had a $36 \%$ lower incidence of breast cancer recurrence compared with those treated with hormonal therapy alone. Unfortunately, the subsequent AZURE (Adjuvant Zoledronic Acid to Reduce Recurrence) trial, performed in a larger number of higherrisk, early-stage breast cancer patients with stage II and III disease treated predominantly with chemotherapy alone versus chemotherapy plus higher intensity zoledronic acid, failed to show a similar improvement in breast cancer recurrence. ${ }^{44}$ However, in subgroup analysis, there was an improvement in both disease-free and overall survival in older $(>5$ years postmenopausal) women treated with zoledronic acid.

There are data to suggest that RANKL may also be integral to the spread and propagation of cancer cells in bone. ${ }^{45}$ The Phase III D-CARE trial is underway to assess the effect of denosumab on disease recurrence in patients with stage II and III high-risk, early-stage breast cancer. ${ }^{46}$ It is hypothesized that denosumab may have the potential to interrupt the "vicious 
Table 3 Considerations for clinical decision-making

\begin{tabular}{|c|c|}
\hline Supporting denosumab use & Supporting IV bisphosphonate use \\
\hline Improved efficacy & Lower risk hypocalcemia \\
\hline Better tolerability & Safer in patients unlikely to be compliant with oral vitamin $D$ \\
\hline Longer time to development of moderate or severe bone pain & and calcium supplements \\
\hline (better prevention of pain) & Possibly lower risk ONJ \\
\hline Safer in patients with progressive or baseline renal insufficiency & Lower cost \\
\hline - Patients receiving nephrotoxic chemotherapy, ie, platinum chemotherapies & Greater than 10 years of experience with use in the medical \\
\hline - Patients with diseases more susceptible to renal dysfunction (ie, prostate cancer) & community \\
\hline \multicolumn{2}{|l|}{ Patients with intolerance to bisphosphonates } \\
\hline \multicolumn{2}{|l|}{ Patients currently receiving an IV bisphosphonate who experience an SRE } \\
\hline \multicolumn{2}{|l|}{ Patients with elevated urinary NTx level despite bisphosphonate therapy } \\
\hline \multicolumn{2}{|l|}{ Ease of subcutaneous administration } \\
\hline \multicolumn{2}{|l|}{ - Patients without port-a-cath or easy IV access } \\
\hline - Patients not receiving monthly chemotherapy infusion & \\
\hline
\end{tabular}

Abbreviations: IV, intravenous; SRE, skeletal-related events; NTx, collagen type I crosslinked N-telopeptide; ONJ, osteonecrosis of the jaw.

cycle" of cancer-induced bone destruction and tumor cell expansion as well as play a pivotal role in breast cancer stem cell survival. ${ }^{47}$ Denosumab has recently shown promising results in preventing bone metastases in prostate cancer. Initial results from the double-blind, placebo-controlled Phase III trial in 147 patients with castrate-resistant prostate cancer at high risk for development of bone metastasis (prostatespecific antigen $>8.0$ or doubling time $<10.0$ months) were recently published. ${ }^{48}$ Seven hundred and sixteen men were randomized to receive denosumab $120 \mathrm{mg}$ subcutaneously every 4 weeks, with a similar number randomized to subcutaneous placebo. Bone metastasis-free survival time (the primary endpoint) was 25.2 months in the placebo group compared with 29.5 months in the denosumab group (HR $0.85,95 \%$ CI $0.73-0.98, P=0.028)$. Overall survival was similar between the two groups. The full results from this trial and those from the D-CARE trial will help define the role of denosumab therapy for preventing cancer progression and specifically bone metastases in patients with prostate and breast cancer.

\section{Conclusion}

Clinicians now have robust and definitive data for the use of the RANKL inhibitor, denosumab, in the treatment and prevention of skeletal-related events in patients with bone metastases from solid tumors. The FDA-approved dose for this indication is denosumab $120 \mathrm{mg}$ subcutaneously every 4 weeks. Other approved options include zoledronic acid $4 \mathrm{mg}$ intravenously every 3-4 weeks and pamidronate $90 \mathrm{mg}$ intravenously every 3-4 weeks. Denosumab has several advantages over bisphosphonate use, including improved efficacy and better tolerability, and offers the convenience of a subcutaneous injection (see Table 3 ). In addition, because denosumab has no known renal toxicity, it may be the preferred choice in patients with baseline renal insufficiency receiving nephrotoxic chemotherapy, or in diseases more susceptible to renal dysfunction, such as renal cell cancer and prostate cancer. However, other toxicities, including osteonecrosis of the jaw and hypocalcemia, are more commonly seen with denosumab than other less potent bisphosphonates (ie, pamidronate) and high-risk patients should be screened and monitored carefully for these toxicities. In addition, the added cost of denosumab as a novel and newly approved biologic agent, particularly with respect to the costs of generic bisphosphonates such as pamidronate, needs to be further evaluated in cost-effectiveness analyses. However, the improvements observed with denosumab compared with zoledronic acid in both pain control, quality of life, and convenience of administration for patients should not be underestimated. Both traditional cytotoxic and targeted chemotherapeutic agents remain essential, because poor control of metastatic disease is still the greatest risk factor for progression of bone disease and the development of skeletal-related events. However, the data presented here highlight the essential role bone-modifying agents such as denosumab play in maintaining the quality of life for patients with bone metastases.

\section{Disclosure}

Dr Stopeck has served in a consultancy or advisory role for and has received honoraria from Amgen Inc (Thousand Oaks, CA) and Novartis Pharmaceuticals (East Hanover, NJ).

\section{References}

1. Coleman RE. Skeletal complications of malignancy. Cancer. 1997; 80(Suppl 8):1588-1594.

2. Coleman RE. The clinical course of bone metastases from breast cancer. Br J Cancer. 1987;55(1):61-66. 
3. Mercandante S. Malignant bone pain: pathophysiology and treatment. Pain. 1997;69(1-2):1-18.

4. Lipton A. Pamidronate prevents skeletal complications and is effective palliative treatment in women with breast carcinoma and osteolytic bone metastases. Cancer. 2000(5);88:1082-1090.

5. Rosen LS. Long-term efficacy and safety of zoledronic acid in the treatment of skeletal metastases in patients with nonsmall cell lung carcinoma and other solid tumors. Cancer. 2004;100(12): 2613-2621.

6. Saad F. Clinical benefits of zoledronic acid for the prevention of skeletal complications in advanced prostate cancer. Clin Prostate Cancer. 2005;4(1):31-37.

7. Hortobagyi GN. Bone metastases in breast cancer patients. Semin Oncol. 1991;18 Suppl 5:11-15.

8. Kohno N. Zoledronic acid significantly reduces skeletal complications compared with placebo in Japanese women with bone metastases from breast cancer: a randomized, placebo-controlled trial. J Clin Oncol. 2005;23(15):3314-3321.

9. Rosen LS. Zoledronic acid is superior to pamidronate for the treatment of bone metastases in breast carcinoma patients with at least on osteolytic lesion. Cancer. 2004;100(1):36-43.

10. Sadd F, Gleason DM, Murray R, et al. A randomized, placebocontrolled trial of zoledronic acid in patients with hormone-refractory metastatic prostate carcinoma. J Natl Cancer Inst. 2002;94(19): $1458-1468$.

11. Rosen LS, Gordon D, Kaminski M, et al. Long-term efficacy and safety of zoledronic acid compared with pamidronate disodium in the treatment of skeletal complications in patients with advanced multiple myeloma or breast carcinoma: a randomized, double-blind, multicenter, comparative trial. Cancer. 2003;98(8):1735-1744.

12. Zometa [package insert]. East Hanover, NJ: Novartis Pharmaceuticals; 2001.

13. Bekker PJ, Holloway DL, Rasmussen AS, et al. A single-dose placebocontrolled study of AMG 162, a fully human monoclonal antibody to RANKL, in postmenopausal women. J Bone Miner Res. 2005;20(12): 2275-2282.

14. Cummings SR, San Martin J, McClung MR, et al. Denosumab for prevention of fractures in postmenopausal women with osteoporosis. N Engl J Med. 2009;361(8):756-765.

15. Brown JP, Prince RL, Deal C, et al. Comparison of the effect of denosumab and alendronate on BMD and biochemical markers of bone turnover in postmenopausal women with low bone mass: a randomized, blinded, phase 3 trial. J Bone Miner Res. 2009;24(1):153-161.

16. Eastell R. Aromatase inhibitors and bone. J Steroid Biochem Mol Biol. 2007;106(1-5):157-161.

17. Hillner BE, Ingle JN, Chlebowski RT, et al. American Society of Clinical Oncology 2003 update on the role of bisphosphonates and bone health issues in women with breast cancer. J Clin Oncol. 2003;21(21): 4042-4057.

18. NCCN Guidelines Version 1.2012 Breast Cancer. 01/20/12. National Comprehensive Cancer Network Inc. Available from: http://www. nccn.org/professionals/physician_gls/pdf/breast.pdf. Accessed February 14, 2012.

19. Ellis GK, Bone HG, Chlebowski R, et al. Randomized trial of denosumab in patients receiving adjuvant aromatase inhibitors for nonmetastatic breast cancer. J Clin Oncol. 2008;26(30):4875-4882.

20. Smith MR, Egerdie B, Hernández Toriz N, et al. Denosumab in men receiving androgen-deprivation therapy for prostate cancer. $N$ Engl J Med. 2009;361(8):745-755.

21. Xgeva ${ }^{\circledR}$ [package insert]. Thousand Oaks, CA: Amgen Inc; 2010.

22. Fizazi K, Lipton A, Mariette X, et al. Randomized Phase II trial of denosumab in patients with one metastases from prostate cancer, breast cancer, or other neoplasms after intravenous bisphosphonates. $J$ Clin Oncol. 2009;27(10):1564-1571.

23. Lipton A, Steger CG, Figueroa J. Randomized active-controlled phase II study of denosumab efficacy and safety in patients with breast cancerrelated bone metastases. J Clin Oncol. 2001;25(28):4431-4437.
24. Tabrizi MA, Tseng CM, Roskos LE, et al. Elimination mechanism of therapeutic monoclonal antibodies. Drug Discov Today. 2006;11(1-2): $81-88$.

25. Kong YY, Yoshida H, Sarosi I, et al. OPGL is a key regulator of osteoclastogenesis, lymphocyte development and lymph-node organogenesis. Nature. 1999;397(6717):315-323.

26. Miller PD, Wagman RB, Peacock M, et al. Effect of denosumab on bone mineral density and biochemical markers of bone turnover: six year results of a phase 2 clinical trial. J Clin Endocrinol Metab. 2011; 96(2):394-402.

27. Stopeck AT, Lipton A, Body JJ, et al. Denosumab compared with zoledronic acid for the treatment of bone metastases in patients with advanced breast cancer: a randomized, double-blinded study. $J$ Clin Oncol. 2010;28(35):5132-5239.

28. Stopeck A, Martin M, Ritchie D, et al. Effect of denosumab versus zoledronic acid treatment in patients with breast cancer and bone metastases: results from the extended blinded treatment phase. Abstract P6-14-01, presented at San Antonio Breast Cancer Symposium, December 8-12, 2010, San Antonio, TX.

29. Stopeck A, Lipton A, Campbell-Baird C, et al. Acute-phase reaction following treatment with zoledronic acid or denosumab: results from a randomized, controlled phase 3 Study in patients with breast cancer and bone metastases. Abstract P6-14-09, presented at San Antonio Breast Cancer Symposium, December 8-12, 2010, San Antonio, TX.

30. Fallowfield L, Patrick D, Body J, et al. Effects of denosumab versus zoledronic acid (ZA) on health-related quality of life (HRQL) in metastatic breast cancer: Results from a randomized phase III trial. Abstract number 1025, presented at the ASCO annual meeting, June 4-8, 2010, Chicago, IL.

31. Van Poznak CH, Temin S, Yee GC, et al. American Society of Clinical Oncology executive summary of the clinical practice guideline update on the role of bone-modifying agents in metastatic breast cancer. $J$ Clin Oncol. 2011;29(16):1221-1227.

32. Fizazi K, Carducci M, Smith M, et al. Denosumab versus zoledronic acid for the treatment of bone metastases in men with castrationresistant prostate cancer: a randomized, double-blind study. Lancet. 2011;377(9768):813-822

33. Brown JE, Cook RJ, Major P, et al. Bone turnover markers as predictors of skeletal complications in prostate cancer, lung cancer, and other solid tumors. J Natl Cancer Inst. 2005;97 (1):59-69.

34. NCCN Guidelines Version 1.2012 Prostate Cancer. 02/22/12. National Comprehensive Cancer Network Inc. Available from: http://www. nccn.org/professionals/physician_gls/pdf/prostate.pdf. Accessed February 14, 2012.

35. Henry DH, Costa L, Goldwasser F, et al. Randomized, double-blind study of denosumab versus zoledronic acid in the treatment of bone metastases in patients with advanced cancer (excluding breast and prostate cancer) or multiple myeloma. J Clin Oncol. 2001;29(9):1125-1132.

36. Richardson GE, Ciuleanu TE, Costa L, et al. Denosumab versus zoledronic acid in patients with bone metastases from solid tumors other than breast and prostate cancers or multiple myeloma: A number needed to treat (NNT) analysis. Abstract number 9115, presented at the ASCO annual meeting, June 3-7, 2011, Chicago, IL.

37. von Moos R, Patrick D, Fallowfield L, et al. Effects of denosumab versus zoledronic acid on pain in patients with advanced cancer (excluding breast and prostate) or multiple myeloma: Results from a randomized phase III clinical trial. Abstract number 9043, presented at the ASCO annual meeting, June 4-8, 2010, Chicago, IL.

38. Lipton A, Fizazi K, Stopeck A, et al. Prevention of skeletal-related events with denosumab or zoledronic acid: combined analysis from 3 registrational trials. Abstract number 3061, presented at the European Multidisciplinary Cancer Congress, September 23-27, 2011, Stockholm, Sweden.

39. Diel IJ, Body JJ, Stopeck A, et al. Effect of denosumab treatment on prevention of hypercalcemia of malignancy in cancer patients with bone metastasis. Abstract number 3051, presented at the European Multidisciplinary Cancer Congress, September 23-27, 2011, Stockholm, Sweden. 
40. Saad F, Brown JE, Van Poznak C, et al. Incidence, risk factors, and outcomes of osteonecrosis of the jaw: integrated analysis from three blinded active controlled phase III trials in cancer patients with bone metastases. Ann Oncol. 2011. Epub Oct 10.

41. Stopeck AT, Lipton A, Martin M, et al. Denosumab in patients with breast cancer and bone metastases previously treated with zoledronic acid or denosumab: results from the 2-year open-label extension treatment phase of a pivotal phase 3 study. Abstract P3-16-07, presented at the San Antonio Breast Cancer Symposium, December 6-10, 2011 , San Antonio, TX.

42. Kendler DL, Roux C, Benhamou CL, et al. Effects of denosumab on bone mineral density and bone turnover in postmenopausal women transitioning from alendronate therapy. J Bone Miner Res. 2010;25(1): 72-81.

43. Gnant M, Mineritsch B, Schippinger W, et al. Endocrine therapy plus zoledronic acid in premenopausal breast cancer. $N$ Engl J Med. 2009;360(22):679-691.
44. Coleman RE, Marshall H, Cameron D. Breast-cancer adjuvant therapy with zoledronic acid. $N$ Engl J Med. 2011;365(15):1397-1405.

45. Jones DH, Nakashima T, Sanchez OH, et al. Regulation of cancer cell migration and bone metastasis by RANKL. Nature. 2006;440(7084): 692-696.

46. Study of denosumab as adjuvant treatment for women with high risk early breast cancer receiving neoadjuvant or adjuvant therapy (D-CARE). Available from: http://clinicaltrials.gov/ct2/show/ NCT01077154. Accessed December 15, 2011.

47. Rodman GD. Mechanisms of bone metastasis. N Engl J Med. 2004; 350(16):1655-1664.

48. Smith M, Saad F, Coleman R, et al. Denosumab and bone-metastasisfree survival in men with castration-resistant prostate cancer: results of a phase 3, randomized, placebo-controlled trial. Lancet. 2012;379(9810): $39-46$.
Biologics: Targets \& Therapy

\section{Publish your work in this journal}

Biologics: Targets \& Therapy is an international, peer-reviewed journal focusing on the patho-physiological rationale for and clinical application of Biologic agents in the management of autoimmune diseases, cancers or other pathologies where a molecular target can be identified. This journal is indexed on PubMed Central, CAS, EMBase, Scopus

\section{Dovepress}

and the Elsevier Bibliographic databases. The manuscript management system is completely online and includes a very quick and fair peerreview system, which is all easy to use. Visit http://www.dovepress. com/testimonials.php to read real quotes from published authors. 